\title{
Automatic Morphology-Based Brain Segmentation (MBRASE) from MRI-T1 Data
}

\author{
Rik Stokking, Koen L. Vincken, and Max A. Viergever \\ I mage Sciences Institute, University Medical Center Utrecht, Room E01.334, Heidelberglaan 100, 3584 CX Utrecht, The Netherlands
}

Received J anuary 31, 2000

\begin{abstract}
A method called morphology-based brain segmentation (MBRASE) has been developed for fully automatic segmentation of the brain from T1-weighted MR image data. The starting point is a supervised segmentation technique, which has proven highly effective and accurate for quantitation and visualization purposes. The proposed method automates the required user interaction, i.e., defining a seed point and a threshold range, and is based on the simple operations thresholding, erosion, and geodesic dilation. The thresholds are detected in a region growing process and are defined by connections of the brain to other tissues. The method is first evaluated on three computer simulated datasets by comparing the automated segmentations with the original distributions. The second evaluation is done on a total of $\mathbf{3 0}$ patient datasets, by comparing the automated segmentations with supervised segmentations carried out by a neuroanatomy expert. The comparison between two binary segmentations is performed both quantitatively and qualitatively. The automated segmentations are found to be accurate and robust. Consequently, the proposed method can be used as a default segmentation for quantitation and visualization of the human brain from T1-weighted MR images in routine clinical procedures. 2000 Academic Press
\end{abstract}

Key Words: segmentation; MRI; brain imaging; mathematical morphology; visualization; image analysis.

\section{INTRODUCTION}

Three-dimensional (3-D) segmentation is the grouping of similar voxels into coherent volumetric structures for quantitative volumetric analysis, morphological analysis, or visualization purposes. An important application area is brain segmentation, e.g., for quantitative analysis of different brain structures (Kohn et al., 1991; Collins et al., 1992; Kikinis et al., 1992), labeling of cortical structures (Sandor and Leahy, 1995; Collins et al., 1995b), localization of electrodes on the surface of the brain (Van den Elsen, 1993), establishing abnormal gyration (Shenton et al., 1992), or providing an anatomical framework for functional studies of the cortex (Stokking et al., 1999).

The most widely applied segmentation technique is manual segmentation, which has several disadvantages: (i) it generally requires a high level of expertise, (ii) it is time and labor consuming, and (iii) it is subjective and therefore not reproducible. Studies investigating inter and intra-patient variations in cerebral function or anatomy have repeatedly shown these shortcomings, which explains the demand for automated techniques (Kikinis et al., 1992; Gerig et al., 1992; Evans et al., 1995; Dale et al., 1999; Lemieux et al., 1999). We focus on a supervised method, originally proposed by Höhne and Hanson (1992), based on region growing and morphological operations. The aim of this paper is to automate the remaining user interaction in this established method, thereby obtaining a fully automated segmentation of the brain, and to evaluate the segmentation results for visualization and volume estimation purposes. We will first give an overview of existing region growing based techniques in the context of MRI brain segmentation followed by a more specific introduction to our segmentation approach.

In this paper we deal with segmentation of MR brain images. In general, simple automatic segmentation techniques fail dramatically because of two disturbing factors; (i) the MR acquisition suffers from image gradients and/or RF coil inhomogeneities, and (ii) different anatomical structures are often linked to each other owing to partial volume effects, noise, imaging artefacts, or by connecting tissue (the optic nerves, blood vessels, etc.). The former factor can be compensated for to some extent by reducing the non-uniformity of the intensities (Wells III et al., 1996; Velthuizen et al., 1998; Sled et al., 1998). To disconnect unwanted anatomical links, the use of morphological operations (erosion/dilation) was found to be effective (Höhne and Hanson, 1992).

MRI segmentation techniques can be roughly divided into three classes: Clustering methods, regionbased methods, and edge-based methods. For a review on segmentation of MRI data we refer to Clarke et al. 
(1995) and Niessen (1997). In this paper we focus on region-based methods with region growing as the main approach.

In region growing-based techniques a segment is formed by selecting a seed pixel and continuously adding neighboring pixels that meet certain-generally simple-requirements, e.g., not exceeding an intensity range. Region growing has the advantage over thresholding that the resulting objects will be spatially connected.

In a classical paper, Zucker (1976) presented an overview of several merge criteria for region growing that were proposed in the early literature on this technique. A more recent comparison of different approaches has been given by J iang et al. (1993). Several techniques have been reported for the use of region growing in medical images. Seeded region growing (Adams and Bischof, 1994; J ustice et al., 1997) makes use of statistical analysis on specified voxels to steer the region growing. Chang and Li (1994) used a region growing process where the thresholds are dynamically and automatically computed using feature histogram analysis.

In general, region growing methods suffer from the fact that the tissue under consideration is readily connected to another tissue type, because of overlapping intensity spectra. Extending region growing with approaches from mathematical morphology (erosion/dilation) has proven effective (Höhne and Hanson, 1992). Others have used a region growing method combined with a connectivity threshold (Barillot et al., 1991) or interactive, semiautomatic leak removal (Sekiguchi et al., 1994). A slightly different approach to region growing has been reported by Cline et al. (1987) who performed a surface extraction from a user specified seed point on the surface. Weak connecting bridges of a few pixels in width are eliminated.

Although several of these approaches can be considered powerful tools for segmentation, none of them is fully automatic and interaction is required to perform a segmentation task. This usually requires a medical expert to actively control the segmentation process and interactively correct the results. One of the most attractive approaches is the segmentation technique of Höhne and Hanson (1992) (we will refer to this technique as the Höhne approach). It is based on region growing and morphological operations (especially erosion and geodesic dilation, i.e., dilation within a mask volume), and requires a user defined seed point as well as a threshold range.

For the analysis of medical volume data, we have used the Höhne approach incorporated into AN ALYZE (Robb and Hanson, 1996) to segment, e.g., the aorta from CTA (Balm et al., 1997), and the brain from MRI (Stokking, 1998). The method proves to be quite practical for reasons of speed, simplicity, and intuitiveness. The technique first extracts a base volume from a data- set using an operator defined threshold (range), thereby encapsulating the required object. A series of erosions is applied to remove undesirable connections with other structures. Subsequent connection to a user defined seed point and geodesic dilation (to counteract the erosion) results in a segmentation of the required object. A considerable part of the process is automated, thereby achieving a formidable decrease in time and labor consumption compared to manual segmentation. However, the process requires little, yet vital interaction from an experienced user, i.e., a seed point must be selected and a threshold range must be set. Especially the latter is crucial to the success of the process (Zucker, 1976; Chang and Li, 1994), which renders the segmentation results subjective and not reproducible.

In this paper we propose a method called morphology-based brain segmentation (MBRASE) to automate the required user interaction of the Höhne approach for segmentation of the brain from MRI-T1 data. MBRASE first selects a seed point and a starting threshold in a heuristic fashion using histogram information. Then MBRASE investigates a region growing process iterating over thresholds to detect a lower and an upper threshold. We evaluated the method for volume estimation and visualization purposes. First, MBRASE is described and the computer simulated and patient data used for evaluation of the method are presented. Then, an extensive evaluation of MBRASE is conducted where difference and similarity measures between MBRASE results and the reference data are calculated. Furthermore, neuroanatomy experts qualitatively evaluated the MBRASE results. The conclusion of the study is that MBRASE is an accurate and reliable method to segment the brain from T1-weighted MRI data.

\section{METHODS AND MATERIALS}

\section{MBRASE}

The starting point of the MBRASE segmentation method is the histogram of grey values of the MRI data (see Fig. 1 for an example). The first and highest peak of the histogram refers to background voxels and is discarded by setting all voxels below a certain percentage of the maximum grey value to zero. The then highest peak is selected as initial threshold value $T_{\text {start }}$. A volume of possible seed points is defined by thresholding with $\mathrm{T}_{\text {start }}$ and applying two erosions with a 6-voxel structuring element. A spherical search from the middle of the dataset is initiated and the first encountered point in the seed volume is marked as the seed point. This heuristic approach for selection of a seed point and $\mathrm{T}_{\text {start }}$ was tested on MRI-T1 datasets of three patients that were not used for final evaluation of MBRASE. 


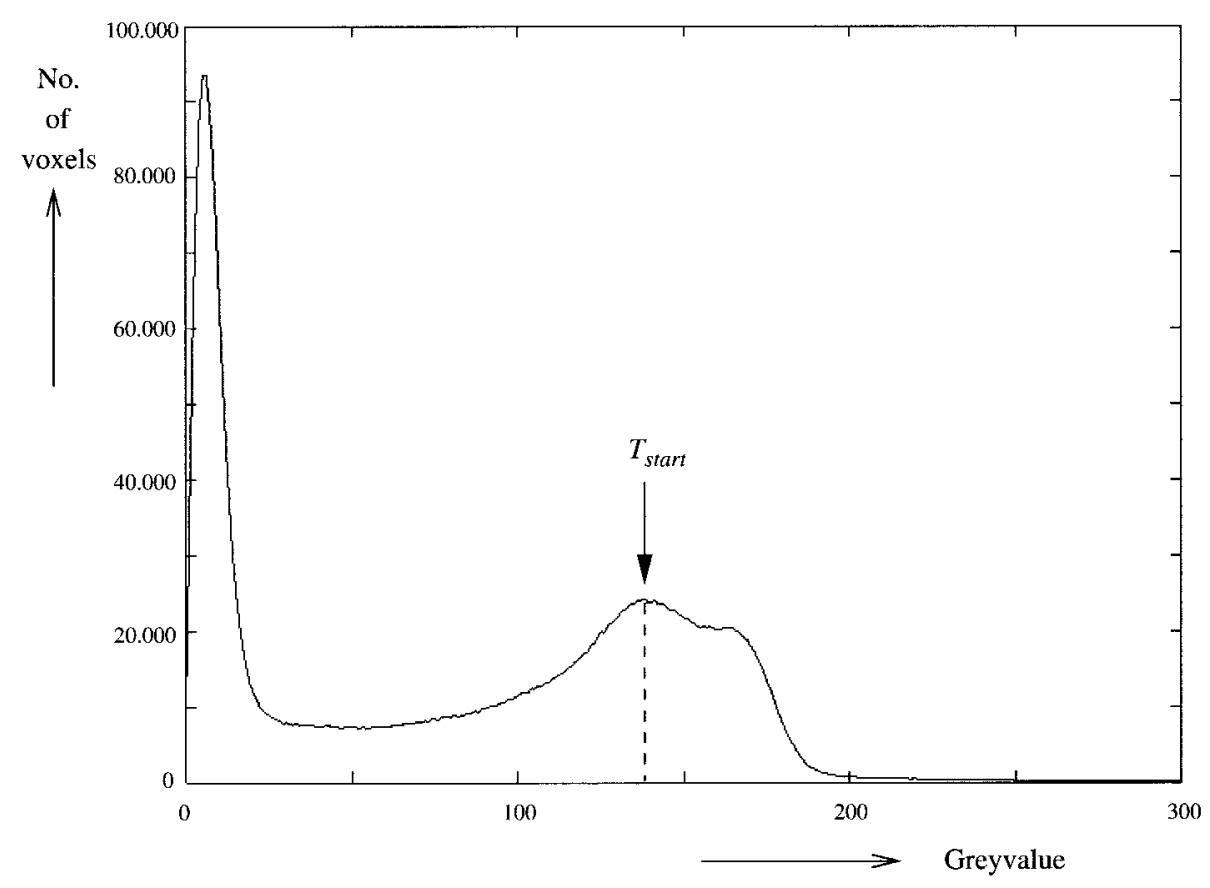

FIG. 1. The histogram of a patient dataset. $T_{\text {start }}$ is defined by the highest value after discarding the background voxels. This value is used as the starting value for the detection of $\mathrm{T}_{\text {low }}$ and $\mathrm{T}_{\text {up. }}$.

The seed point and $\mathrm{T}_{\text {start }}$ are the input values for the next and most important component of MBRASE, i.e., the definition of the threshold range. This process consists of two parts that iterate over threshold settings. The first part begins at $\mathrm{T}_{\text {start }}$ and with each iteration of the process the threshold is decreased by a fixed number (a step size of 1 is generally recommended) until the lower threshold ( $\left.T_{\text {low }}\right)$ is detected (see Fig. 2$)$. The second part of the process detects the upper threshold $\mathrm{T}_{\text {up }}$ by starting at $\mathrm{T}_{\text {start }}$ and increasing the threshold at each iteration. Since $T_{\text {low }}$ is already determined, this information is used in the latter part of the process to facilitate and optimize the search for $T_{\text {up }}$.

Both parts of the process for threshold detection iterate over threshold settings. At each threshold setting four steps are executed: (I) thresholding, (II) erosion, (III) region growing, and (IV) peak detection. When no peak is detected in step IV, the threshold is decreased (for $T_{\text {low }}$ ) or increased (for $T_{\text {up }}$ ) and the four steps are repeated for the new threshold setting using the current region growing volume as the seed volume. This continues until a peak is detected which finishes the search for $\mathrm{T}_{\text {low }}$ or $\mathrm{T}_{\text {up }}$. The peak typically signals the inclusion of a new, sizable nonbrain segment through a connection. The previous threshold value then defines $\mathrm{T}_{\text {low }}$ or $\mathrm{T}_{\text {up, }}$, because the inclusion of a new segment is to be avoided.

Although the 4 steps are basic operations, some additional explanation is required. In step I thresholding is applied to extract a base volume from the grey value data. This base volume is eroded twice (step II) with a 6-voxel structuring element to break small connections. A region growing procedure (step III) is initiated from the seed point or current seed volume, and grows one layer of voxels at each iteration of this region growing process with the eroded base volume as a

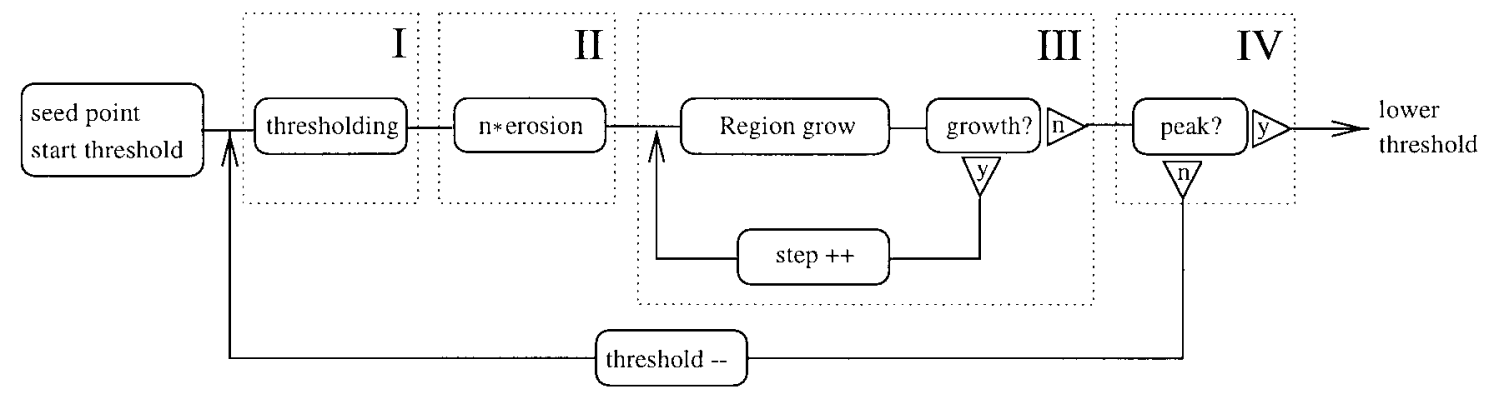

FIG. 2. Schematic of the algorithm for detection of the lower threshold $\mathrm{T}_{\text {low }}$. 

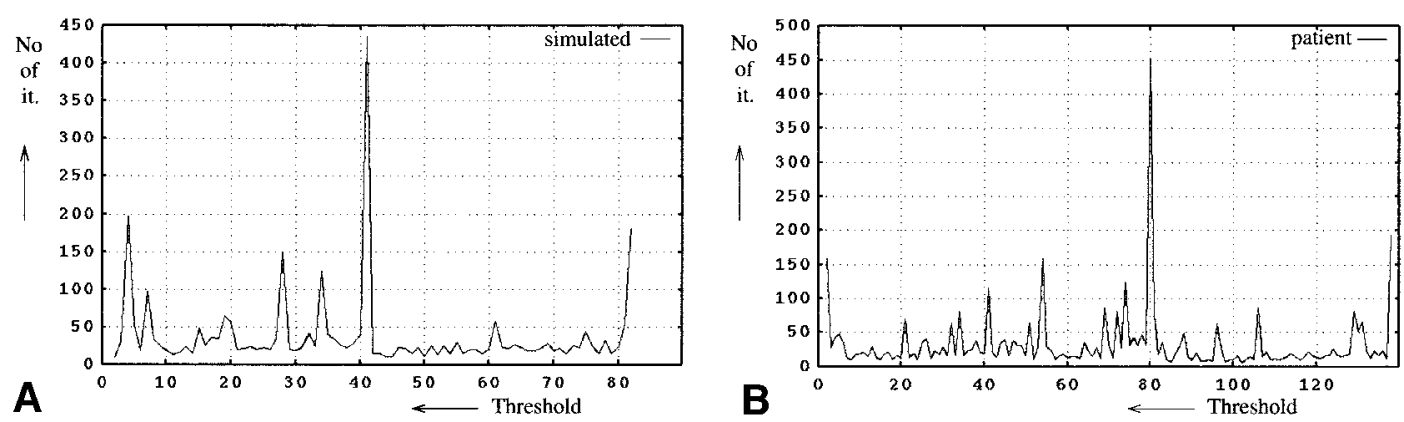

FIG. 3. Detection of $T_{\text {low. }}$ The number of iterations (No of it.) with a decreasing threshold value for a computer simulated (A) and a typical patient (B) dataset are presented. The iteration process for the simulated data started with a $\mathrm{T}_{\text {start }}$ of 82 . The peak at value 41 was detected resulting in $\mathrm{a} \mathrm{T}_{\text {low }}$ of 42 . The process for the patient data started at 139 . The peak at value 80 was detected resulting in a $\mathrm{T}_{\text {low }}$ of 81 . F or both datasets the increases in the number of iterations earlier on in both processes are not significant enough to qualify as a peak. For results of the corresponding segmentations see Fig. 4 for the simulated dataset and Fig. 5 for the patient dataset.

mask (i.e., no growing of the volume outside the eroded base volume is permitted). Eventually the growing stops, yielding the region growing volume corresponding to the current threshold. Step IV is a peak detection step to determine whether a new, sizable segment is included in the region growing volume through a connection. A simple comparison is performed between the total number of iterations of the region growing process for the current threshold and the number of iterations at the previous threshold settings. This last step requires several parameters and will be explained first.

MBRASE was initially tested and optimized using three patient datasets. The acquisition protocol for these three sets was comparable to the protocol used for the group of 30 in our final analysis. Based on the tests, we decided to define the current number of iterations as a peak whenever this number exceeds 1.5 times the sum of the iterations at the previous five threshold settings. Unfortunately, no $T_{\text {up }}$ was detected in two of the three test-cases as the brain did not connect to other structures when increasing the threshold from $T_{\text {start }}$. In these cases no peak was detected and the process did not stop. We therefore added an extra stopping criterium: The process for the detection of $\mathrm{T}_{\text {up }}$ stops whenever the number of iterations in the region growing step is zero for five consecutive threshold settings, i.e., no growth occurs.

To illustrate the peak detection process we will focus on the detection of $T_{\text {low. }}$. Figure 3 shows the typical results of the region growing process for a computer simulated dataset and a patient dataset (see next subsection). The process for the simulated data begins with a $T_{\text {start }}$ of 82 and the patient data begins at a $T_{\text {start }}$ of 139. Normally, the process terminates once a peak is detected, but for illustration purposes peak detection is turned off and the process continues until the threshold setting reaches the value one. For both the simulated and the patient study the detected peaks-at values 41 (simulated) and 80 (patient) - signal the inclusion of a sizable nonbrain segment as can be verified in Fig. 4 for the simulated data and in Fig. 5 for the patient data (see Results). The previous threshold setting is then selected as $T_{\text {low }}$, i.e., 42 for the computer simulated data and 81 for the patient data. Note that the small increases between $\mathrm{T}_{\text {start }}$ and the detected peak did not meet the peak detection criteria.
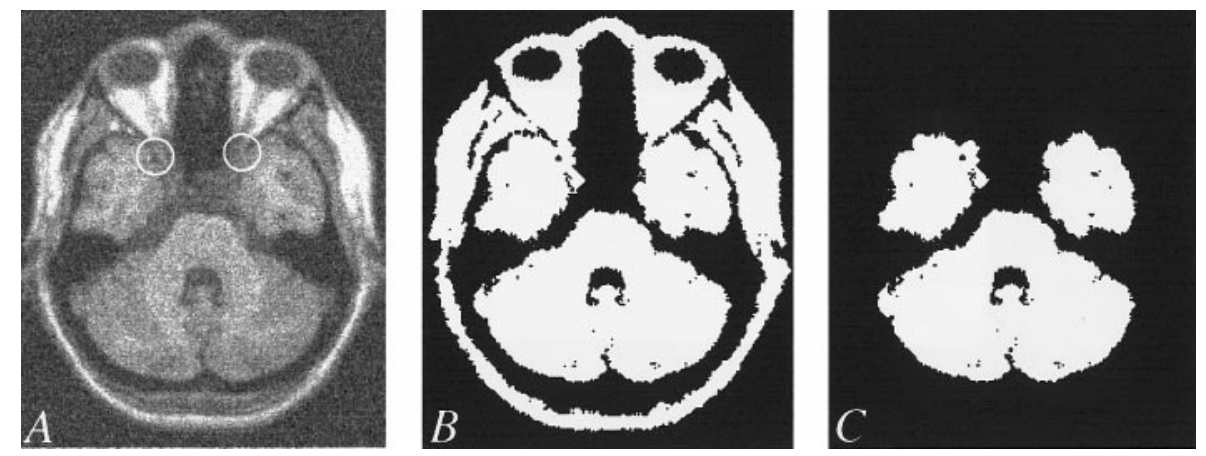

FIG. 4. Intermediate results of the MBRASE method for simulated MRI data with extreme noise and RF inhomogeneity (9n40RF). A representative slice of the original grey value data is shown in Frame (A). Frame (B) is the result when applying a $T_{\text {low }}$ of 41 (see $F$ ig. $3 A$ ) for segmentation of these grey value data. Frame (C) presents the segmentation result with a $T_{\text {low }}$ of 42 showing the exclusion of the undesired segment(s). Frame (C) is also the final result of the MBRASE method, since no $T_{\text {up }}$ was detected. The circles in Frame (A) indicate the relatively low grey value connections that define $\mathrm{T}_{\text {low. }}$. 

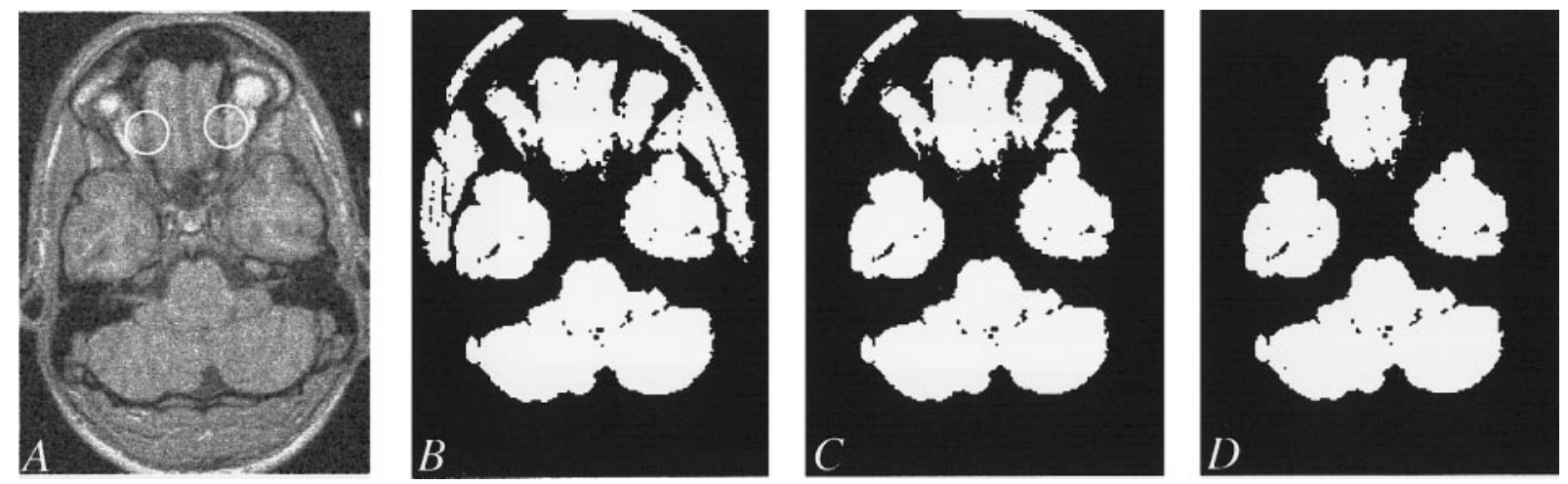

FIG. 5. Intermediate results of the MBRASE method for a patient set. A representative slice of the original grey value data is shown in Frame (A). Frame (B) is the result when applying a $\mathrm{T}_{\text {low }}$ of 80 (see Fig. 3B) for segmentation of these grey value data. $\mathrm{Frame}(\mathrm{C})$ presents the segmentation result with $\mathrm{T}_{\text {low }}$ of 81 showing the exclusion of the undesired segment(s). Frame (D) is the final result using both $\mathrm{T}_{\text {low }}(81)$ and $\mathrm{T}_{\text {up }}$ (224). Here, differences between Frames (C) and (D) show the importance of the upper threshold to break the connections owing to relatively high grey values. The circles in Frame $(A)$ indicate the high grey value connections that define $T_{\text {up }}$.

An important step in the process is the use of erosions with each threshold before growth is permitted. This effectively removes small connections caused by anatomy and the partial volume effects and thus ensures a more dramatic increase in the number of iterations growth per threshold when a new segment is incorporated.

Apart from the parameters of the peak detection step, MBRASE requires a set of parameters for the morphological operations. These parameters for the region growing, erosion, and dilation processes are set to two erosions, three (is number of erosions plus one) geodesic dilations and a 6-voxel structuring element. This is based on information from the ANALYZE reference manual and our experience with segmentation procedures.

The final segmentation is performed by applying the seed point and the threshold range $\left[\mathrm{T}_{\text {low }}-\mathrm{T}_{\text {up }}\right]$ for segmentation of the brain using the Höhne approach.

\section{Computer-Simulated and Patient Data}

MRI computer simulations of a head phantom dataset have been made available by the McConnell Brain I maging Centre at the Montreal Neurological I nstitute (http://www.bic.mni.mcgill.ca/brainweb). The head phantom is described in (Collins et al., 1998) and the simulator in (Kwan et al., 1999). Each voxel of the phantom can contain multiple tissue types, thus providing a fuzzy (i.e., partial volume voxel) model (Cocosco et al., 1997). To simplify the comparison between segmentation results we binarize the Montreal phantom by selecting all voxels with a probability for brain (denoted grey, white, and glial matter in the Montreal phantom dataset) higher than or equal to $50 \%$ (see also Vincken et al., 1998).

MRI brain datasets can be simulated using differences in pulse sequence, slice thickness, noise (options: $0,3,5,7$, and $9 \%$ of the standard deviation of the mean intensity of white matter) and nonuniformity (RF) (options: 0,20 , and $40 \%$ resulting in a (varying) $\mathrm{RF}$ field of: 1, 0.9-1.1, and 0.8-1.2). Three MRI-T1 datasets with a slice thickness of $1 \mathrm{~mm}$ are used; (i) 3\% noise and an RF of $0 \%$ (simulated data code: 3n00RF), (ii) 3\% noise and an RF of 20\% (3n20RF), and (iii) $9 \%$ noise and an RF of $40 \%$ (9n40RF). Note: $3 \%$ noise and an RF of $20 \%$ are denoted typical, while $9 \%$ noise and an RF of $40 \%$ are extreme (Cocosco et al., 1997).

The patient data are T1-weighted 3-D gradient-echo MR scans (voxel size $1 \times 1 \times 1.2 \mathrm{~mm}$ ) of 30 patients (age range 6 to 17 years) from the Department of Child Psychiatry at our University Hospital diagnosed with the Gilles de la Tourette Syndrome, Attention-Deficit Hyperactivity Disorder, autistic behavior, and/or Obsessive Compulsive Disorder. Upon initial screening, no gross abnormalities were detected in the MRI data.

\section{Evaluation Methodology}

The evaluation of the simulated studies can be done in an objective fashion, since the segmentation obtained with MBRASE can be compared with the binarized distribution of the computer phantom. This

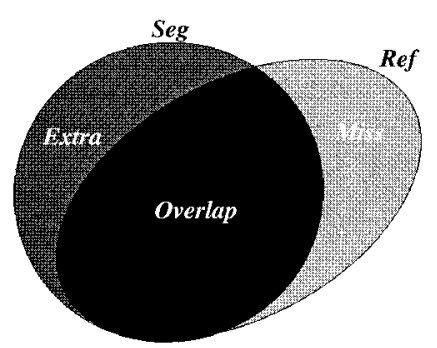

$$
\begin{aligned}
\% O & =\frac{\text { Overlap }}{\text { Ref }} \times 100 \% \\
\% E & =\frac{\text { Extra }}{\text { Ref }} \times 100 \% \\
S I & =\frac{2 \times \text { Overlap }}{\text { Seg }+ \text { Ref }}
\end{aligned}
$$

FIG. 6. Three measures, i.e., percentage overlap $(\% \mathrm{O})$, percentage extra (\%E), and the Similarity Index (SI), are calculated to compare a binary segmentation set (Seg) with the corresponding binary reference dataset (Ref). 
TABLE 1

Measures for the Computer Simulated Data

\begin{tabular}{clcc}
\hline Simulated & $\% \mathrm{O}$ & $\% \mathrm{E}$ & $\mathrm{SI}$ \\
\hline 3nOORF & $99.7 \%$ & $6.4 \%$ & 0.964 \\
3n2ORF & 97.8 & 1.4 & 0.982 \\
9n40RF & 99.6 & 11.4 & 0.944 \\
\hline
\end{tabular}

Note. Results for the three simulated datasets with respect to the binarized phantom segmentation (see also Fig. 6).

reference set is called the binarized phantom segmentation.

With patient data an objective gold standard is not available. However, we had already segmented the patient datasets for visualization purposes using the supervised method of Höhne in an earlier project (Stokking et al., 1999) and we decided to use these segmentation results as reference sets for comparison. We will refer to them as supervised patient segmentations.

In order to quantitatively assess the correspondence between a binary segmentation set and the corresponding binary reference set, we applied three mea- sures, \%O (percentage overlap), \%E (percentage extra), and the Similarity Index (SI) (Zijdenbos et al., 1994) (see Fig. 6). \%O equals the number of voxels segmented by MBRASE that overlap with the reference segmentation, divided by the total number of voxels in the reference dataset. \%E equals the fraction of voxels segmented by MBRASE that are not in the reference segmentation. SI is a measure derived from kappa statistics. It expresses the number of voxels overlap divided by the total number of segmented voxels in both files and is therefore sensitive to both differences in size and location. The factor of 2 ensures that a perfect segmentation has a similarity value of 1 .

The qualitative visual inspection of the visualization results was performed by three neuroanatomy experts. Typical examples of the investigated 2-D and 3-D visualizations are shown under Results.

\section{RESULTS}

The parameter $\mathrm{T}_{\text {start }}$ and the seed point are extracted from the histogram and the data as described under Methods and Materials.
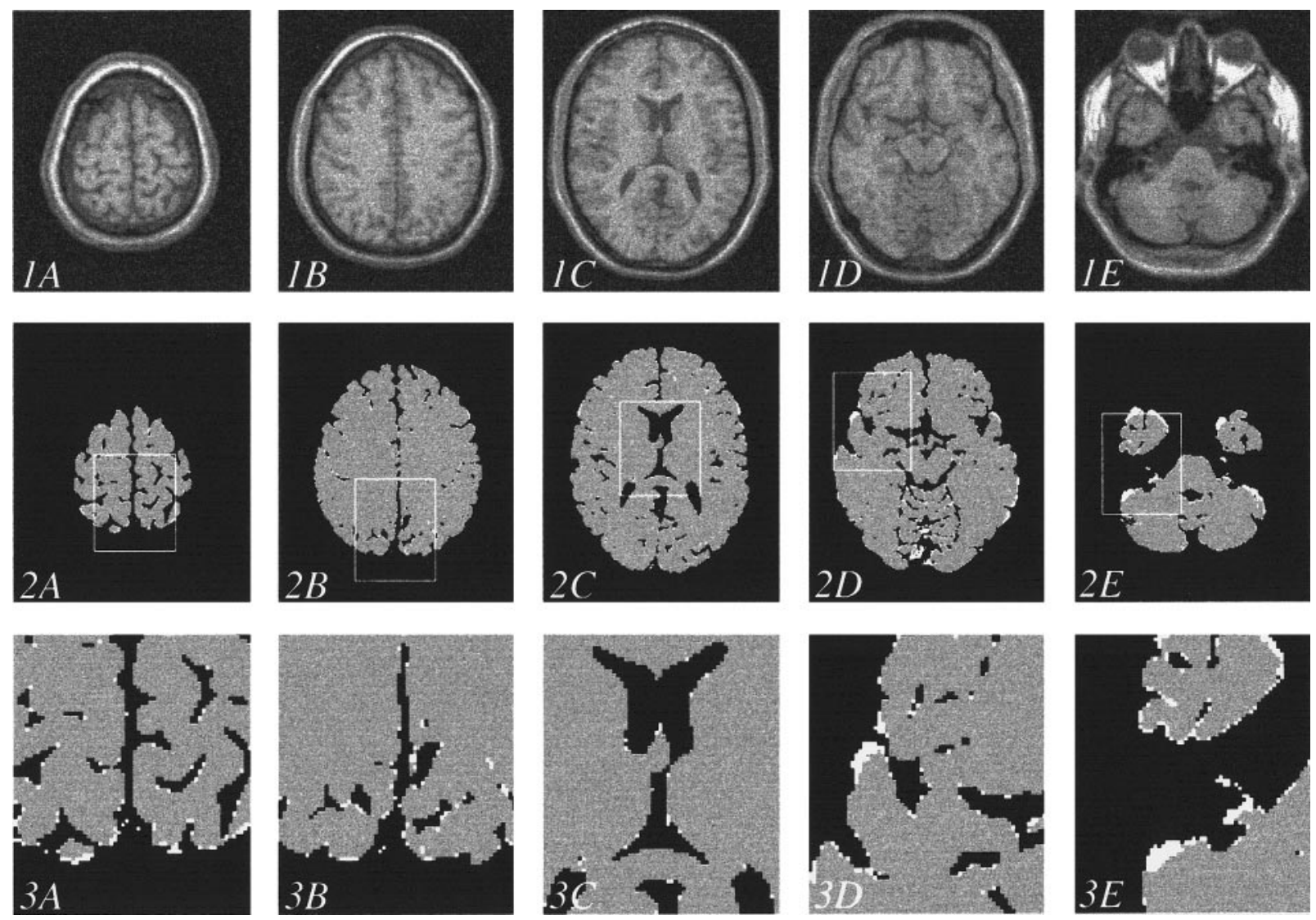

FIG. 7. Results of the MBRASE method for the realistic simulated set (3n20RF) compared with the binarized phantom segmentation. Top row: Representative slices of the original grey value data for 3n20RF. The second row presents in grey the overlap between the MBRASE result and the binarized phantom segmentation. The differences are shown in white. The third row shows enlargements of the indicated details in the second row. 

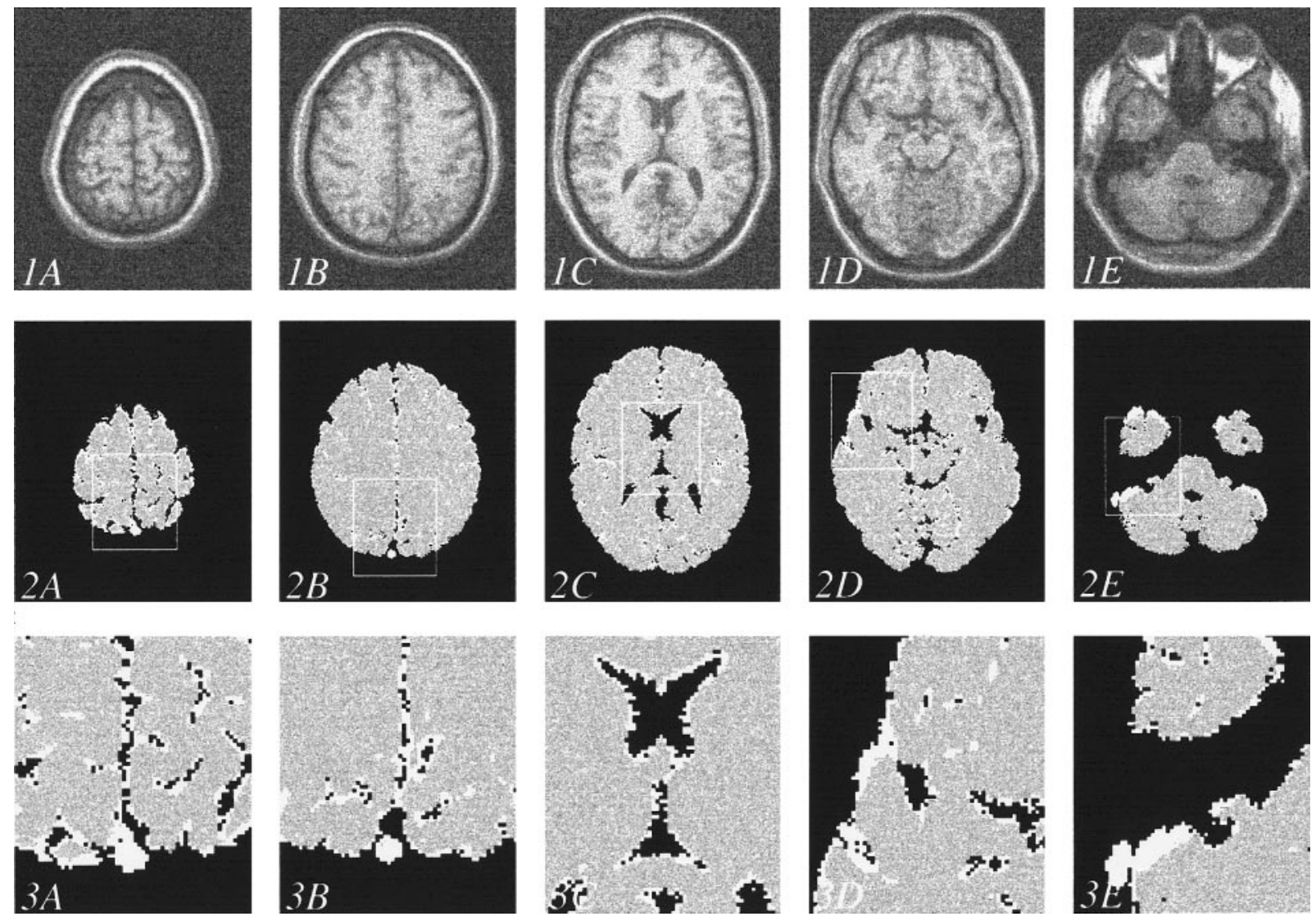

FIG. 8. Results of the MBRASE method for the simulated set with the highest level of noise and RF inhomogeneity (9n40RF) compared with the binarized phantom segmentation. See legend of Fig. 7 for set-up.

The results of the comparison of MBRASE with the simulated and patient datasets are illustrated by highlighting both the extra and missing voxels (see Fig. 6). In most datasets, the differences mainly concern voxels that were segmented extra by MBRASE.

\section{Simulated Data}

Table 1 shows the measures of MBRASE for the three simulated sets compared with the binarized phantom segmentation. Similarities are high, especially for the realistic simulated dataset 3n20RF. We present the resulting images for two simulated sets, i.e., the set with the realistic levels of noise and RF inhomogeneities ( $3 n 20 R F)$ and the set with the extreme levels of noise and RF inhomogeneities (9n40RF) (see also Fig. 4 for intermediate results).

The results for the simulated set with the realistic settings (3n20RF) are displayed in Fig. 7. The differences of the MBRASE segmentations with the ground truth are very small.

The results for the simulated dataset with the extreme values of noise and RF nonuniformity are shown in Fig. 8. Here, the differences are largely due to voxels segmented extra by MBRASE. Although the similarity index for this dataset is the lowest of all segmentations, including patient cases, the images show that the visual similarity between the MBRASE segmentation and its reference can still be considered high.

The results for all three simulated sets indicated two major problem areas for the MBRASE segmentation: (i) The inclusion of vessels and meninges, especially in the longitudinal cerebral fissure. (ii) The exclusion of thin, long brain structures, especially in parts of the cerebellum.

\section{Patient Data}

In Table 2 the measures for the 30 patient datasets are presented. With 28 of these sets, MBRASE successfully segmented the brain using the default peak detection level of 1.5. For the other two datasets (patient sets XIV and XXIII) the initial measures were not consistent with the rest of the patient sets. Investigation revealed that no peak was detected in case XIV, whereas in case XXIII the first peak was missed. This situation could be automatically flagged and remedied by a single change of parameter: reduction of the peak detection level from 1.5 to 1.1. In Table 2 the results of 
TABLE 2

Measures for the Patient Data

\begin{tabular}{|c|c|c|c|}
\hline Patient & $\%$ & $\% \mathrm{E}$ & SI \\
\hline I & $100.0 \%$ & $6.8 \%$ & 0.966 \\
\hline II & 99.4 & 0.1 & 0.996 \\
\hline III & 100.0 & 8.4 & 0.959 \\
\hline IV & 99.7 & 2.5 & 0.986 \\
\hline V & 100.0 & 3.2 & 0.984 \\
\hline VI & 100.0 & 8.6 & 0.958 \\
\hline VII & 99.9 & 4.4 & 0.978 \\
\hline VIII & 100.0 & 4.8 & 0.976 \\
\hline IX & 99.9 & 5.1 & 0.974 \\
\hline$x$ & 99.5 & 1.4 & 0.990 \\
\hline XI & 100.0 & 5.0 & 0.975 \\
\hline XII & 100.0 & 11.7 & 0.944 \\
\hline XIII & 99.3 & 0.0 & 0.996 \\
\hline XIV* & 99.1 & 5.5 & 0.968 \\
\hline$X V$ & 100.0 & 8.3 & 0.959 \\
\hline XVI & 96.2 & 0.0 & 0.985 \\
\hline XVII & 96.5 & 0.1 & 0.981 \\
\hline XVIII & 100.0 & 2.9 & 0.985 \\
\hline XIX & 99.8 & 2.8 & 0.984 \\
\hline$X X$ & 97.5 & 0.3 & 0.985 \\
\hline XXI & 100.0 & 4.2 & 0.979 \\
\hline XXII & 99.8 & 5.4 & 0.972 \\
\hline XXIII* & 100.0 & 3.0 & 0.985 \\
\hline XXIV & 100.0 & 0.7 & 0.996 \\
\hline XXV & 100.0 & 2.3 & 0.988 \\
\hline XXVI & 100.0 & 3.6 & 0.982 \\
\hline XXVII & 100.0 & 2.2 & 0.989 \\
\hline XXVIII & 99.6 & 1.0 & 0.993 \\
\hline XXIX & 99.8 & 0.1 & 0.998 \\
\hline XXX & 98.8 & 0.2 & 0.993 \\
\hline
\end{tabular}

Note Results for the patient datasets (see also Table 1 and Fig. 6). The results for the two patients indicated with an asterisk were included after a change of parameter (see the text for details).

these two studies are presented after the change of parameters.

For the 28 sets the measures $\% \mathrm{O}$ and $\% \mathrm{E}$ indicate that with almost all of these sets MBRASE segments extra tissue surrounding the brain when compared to the corresponding reference sets. Furthermore, the similarity measures of these sets are high (average of 0.980 with a standard deviation of 0.013 ). We selected three datasets for presentation of the results, i.e., the sets with the lowest (XII), average (XXI, see also Fig. $5)$, and highest (XXIX) similarity.

The differences in the segmentations between the MBRASE method and the supervised (Höhne) method for case XII (lowest similarity) are shown in Fig. 9. The differences are most conspicuous in the vessels (especially the sinuses) and meninges on the surface of the brain (see also the renderings in Fig. 10).

For cases XXI (average similarity, see Fig. 11) and $X X I X$ (highest similarity, see Fig. 12), the differences in the segmentations are small. Visual comparison between the renderings of the MBRASE segmentation results and the supervised segmentation results using the Höhne approach showed that the differences were hardly noticeable. We therefore only show the renderings of the MBRASE segmentation results (see Fig. 13).

\section{DISCUSSION}

We used several white matter voxels in different parts of the brain as seed points for the region growing process so as to test the location dependency of the seed point. The location only affected the beginning of the growth graph of the process; the graphs of the different seed points rapidly showed the same pattern leading to identical values for $T_{\text {low }}$ and $T_{\text {up }}$.

The applicability of the MBRASE method depends on the sensitivity to the two types of parameters, i.e., the parameters of the morphological operations and the parameters for the peak detection algorithm. Based on the description of the Höhne approach in the ANALYZE manual and our own experiences with MRI-T1 brain data, we use two erosions, three geodesic dilations and a 6-voxel structuring element for all morphological operations. In theory these parameters are influenced by the spatial resolution, (non-) isotropic scanning, noise and RF inhomogeneity. For example, in another project (Stokking et al., 2000) we experienced significant problems segmenting some older MR scans (1994 and earlier), but this was due to the poor quality of the data. Also, severely anisotropic data caused problems because morphological operators work best with isotropic data. As a general rule of thumb, we do not apply the Höhne approach for data where the resolution and anisotropy are worse than $1 \times 1 \times 3 \mathrm{~mm}$. On the other hand, we have applied the Höhne segmentation approach with the aforementioned parameters on high resolution MRI-T1 data acquired using several scanners with several protocols and we obtained adequate segmentation results for all of these types of MRI-T1 data with these parameters.

The critical decision in MBRASE is the detection of the peak in the region growing processes for $T_{\text {low }}$ and $\mathrm{T}_{\text {up }}$. A peak typically signals a connection between the brain and a new, sizable structure and we use a detected peak to define the thresholds for the final segmentation of the brain. Since MBRASE is based on the Höhne approach using two erosions with a 6-voxel structure element, the connection causing the peak is a connection that remains present even after these erosions.

The peak detection performs a simple comparison of the present number of region growing iterations with the numbers at the previous threshold settings. This algorithm and its parameters may have to be adjusted for a different MRI acquisition technique and/or scanner. However, we have shown that this can be done adequately using just a few (here: three) test datasets. Furthermore, we emphasize that MBRASE evaluates 

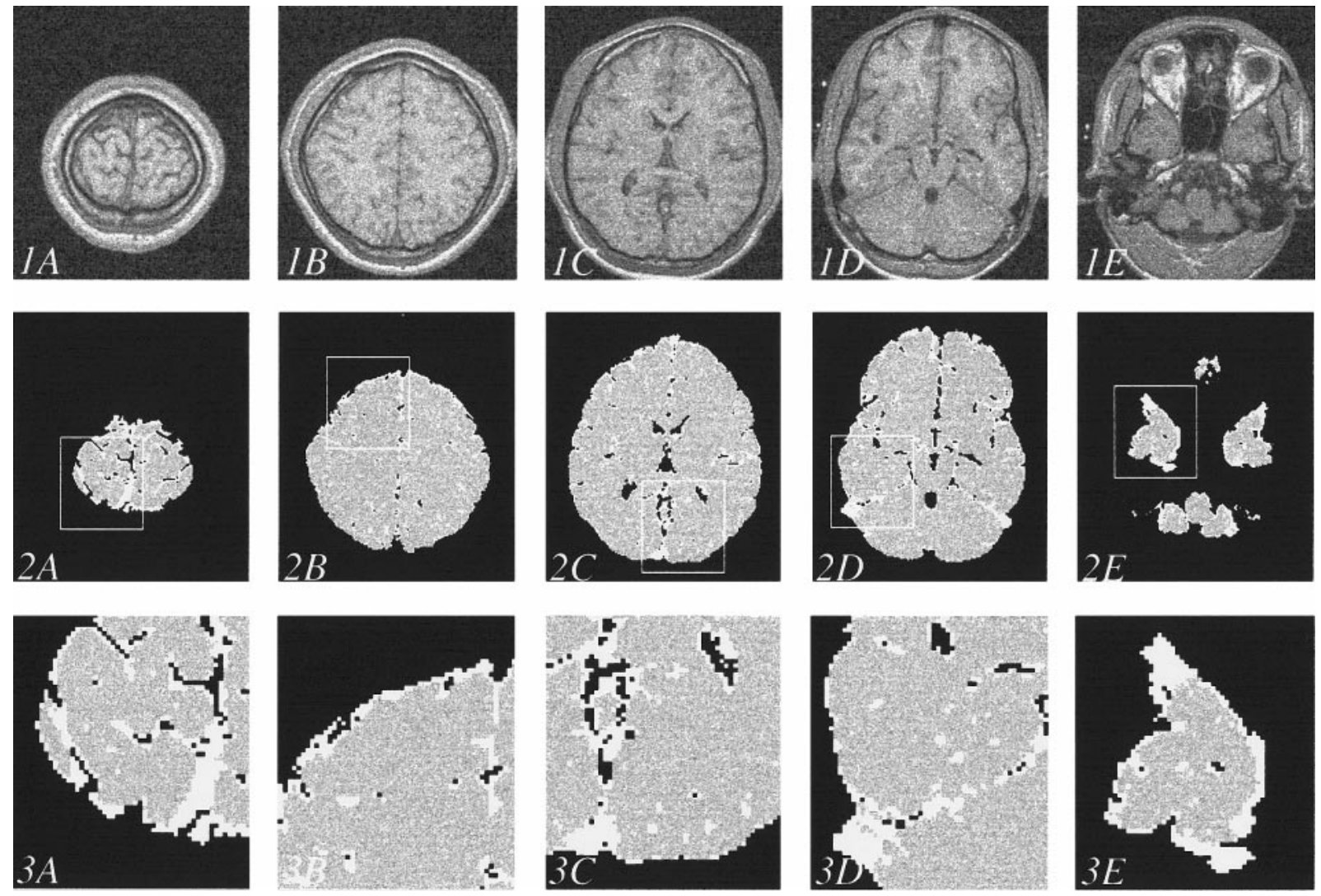

FIG. 9. Results of the MBRASE segmentation compared with the supervised segmentation using the Höhne method for patient dataset XII (lowest similarity). See legend of Fig. 7 for set-up.

the growth in number of iterations, not in number of voxels. While the latter growth can be an indication for the inclusion of a new segment (e.g., used by (Lemieux et al., 1999)), we found the number of iterations a much stronger indicator for MRI-T1 brain data because growth in non-brain structures is typically in long elongated structures like fat or skin. Growth in these structures requires a lot of iterations in the region growing process, but does not necessarily include a lot of voxels.
MBRASE only failed in two cases out of 30 patient studies for the initial parameter settings. Failures are immediately apparent and are easily flagged upon retrospective investigation of the growth graph (see Fig. 3). For the two cases a satisfactory segmentation was obtained by adjusting just one parameter, viz. lowering the peak detection parameter. This lower value resulted in outliers for other cases, so a procedure that works flawless for just one parameter setting has ap-
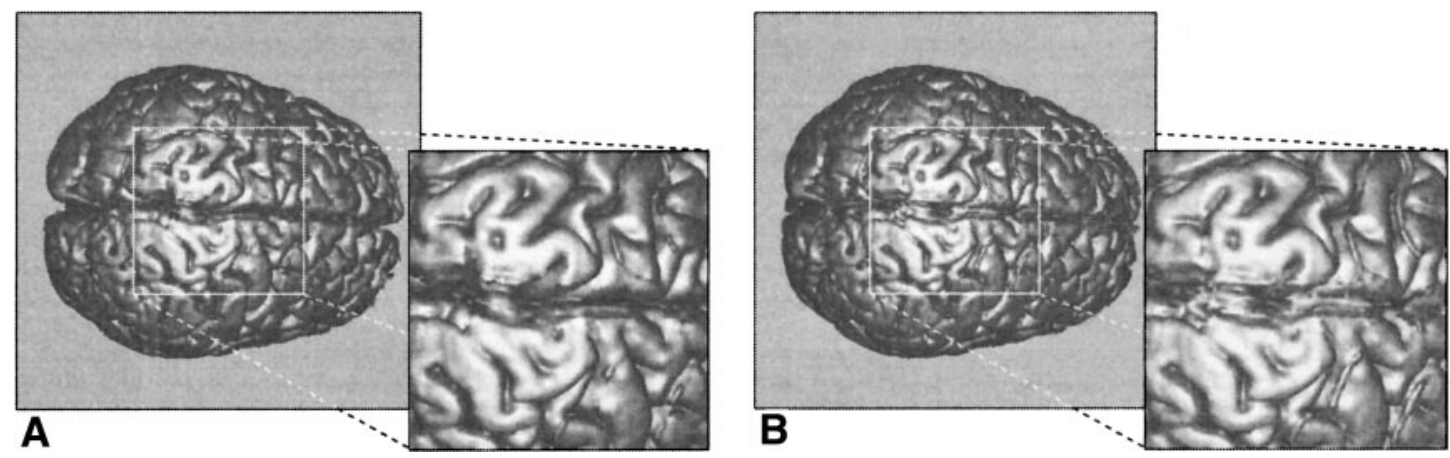

FIG. 10. Comparison of volume renderings for patient dataset XII (lowest similarity) with an enlargement of the longitudinal cerebral fissure. On the left (A) the images resulting from the supervised segmentation using the Höhne method, on the right (B) the corresponding image of the MBRASE method. 

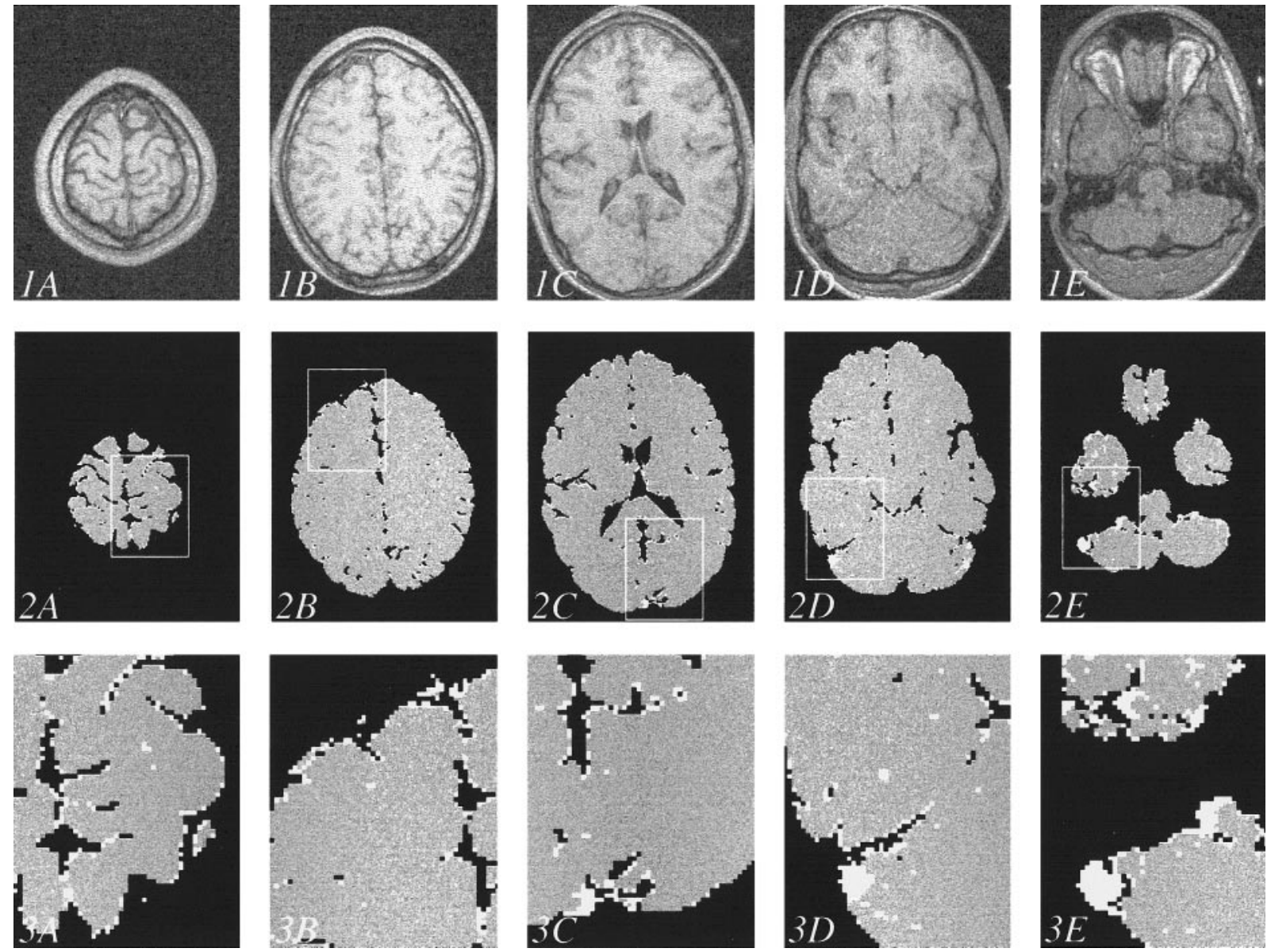

FIG. 11. Results of the MBRASE segmentation compared with the supervised segmentation using the Höhne method for patient dataset XXI (average similarity). See legend of Fig. 7 for set-up.

peared not feasible. We also tested other, more elaborate peak detection methods, e.g., variation analysis, but these methods did not improve the segmentation results compared to the initial peak detection method we applied.

In this study MBRASE was evaluated using the MRI-T1 datasets of pediatric patients and the results may not be completely representative for adult brains. However, preliminary testing of MBRASE on MRI-T1 data of adult brains has indicated that the results are comparable to the results in this report.

In the studies with patient data, the supervised segmentation method of Höhne is used as a reference, not as a gold standard. When comparing both methods, we have to realize that MBRASE is in fact an automated version of the Höhne technique and both will have some identical problems. First of all, we previously mentioned that the Höhne approach is influenced by the spatial resolution, (non-) isotropic scanning, noise, and RF inhomogeneity, and this also applies for MBRASE. Secondly, the application of erosion and subsequent geodesic dilation is a very simple and powerful technique for removal of the (thin) connections be tween different tissues, but it also affects possible long, thin intratissue structures. For instance, the cerebellum manifests some filament-like parts that are easily eroded, but difficult to regain via dilations within a threshold mask (see Fig. 12, frames $1 \mathrm{C}$ and $2 \mathrm{C}$, where part of the cerebellum, i.e., the vermis, is missing in both the reference and MBRASE segmentation).

With the patient datasets the segmentation procedures employed by MBRASE and the expert (using the Höhne approach) have a high similarity, but there are also some distinct differences that account for the (small) differences in the results between the two. The critical decision in MBRASE, i.e., the detection of the peak that defines a threshold, is based only on the first established (3-D) connection between the brain and a new, sizable structure, which cannot be broken by the erosions. With the Höhne approach, the expert qualitatively investigated connections in 2-D images (typically three sets of 10 consecutive MR slices) and had to visually assess whether the (3-D) connections could be broken using the erosions. Also, the connectivity was only one of the criteria used by the expert to determine a threshold range. For example, the overall shape of 

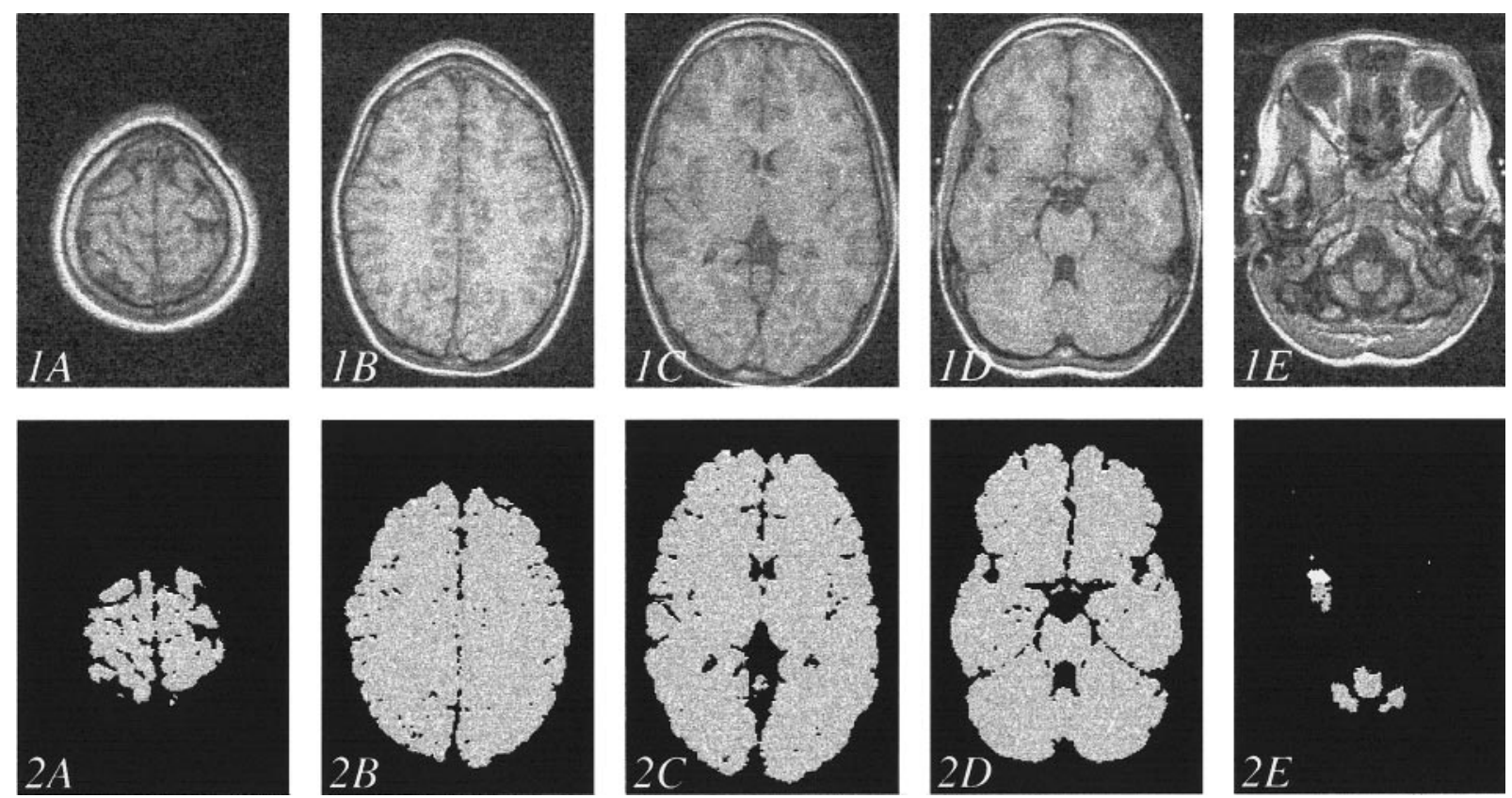

FIG. 12. Results of the MBRASE segmentation compared with the supervised segmentation using the Höhne approach for patient dataset XXIX (highest similarity). The top row shows representative slices of the dataset. The second row presents the overlap between the two sets in grey, the differences in white.

the surface of the brain when using a certain threshold was also an important criterion. Furthermore, with the Höhne approach the operator typically evaluated the results obtained using several threshold settings (especially varying the lower threshold) to find an optimal setting.

The absence of a gold standard for the patient data raises the question whether MBRASE or the expert performed better on the segmentation task. In the majority of patient cases MBRASE appears to segment some additional tissue surrounding the brain. This implies that the expert used a (slightly) higher $\mathrm{T}_{\text {low }}$ compared to MBRASE, which probably stems from the fact that the expert qualitatively investi- gated the connections in 2-D images and also used more criteria. The extra tissue MBRASE segments is considered of little importance, both for quantitation and for visualization purposes. The 2-D images show only small differences. The impact of the extra tissue on the 3-D volume visualizations is even smaller because the rendering package we use (VROOM (Zuiderveld, 1995)) employs a shading algorithm which assigns opacities to the grey value data in a neighborhood of the surface voxel (see also (Levoy, 1988)). This ensures that additional tissue surrounding the brain has only little impact on the final image. Furthermore, the parameters for this shader are automatically set using the detected $\mathrm{T}_{\text {low. }}$.
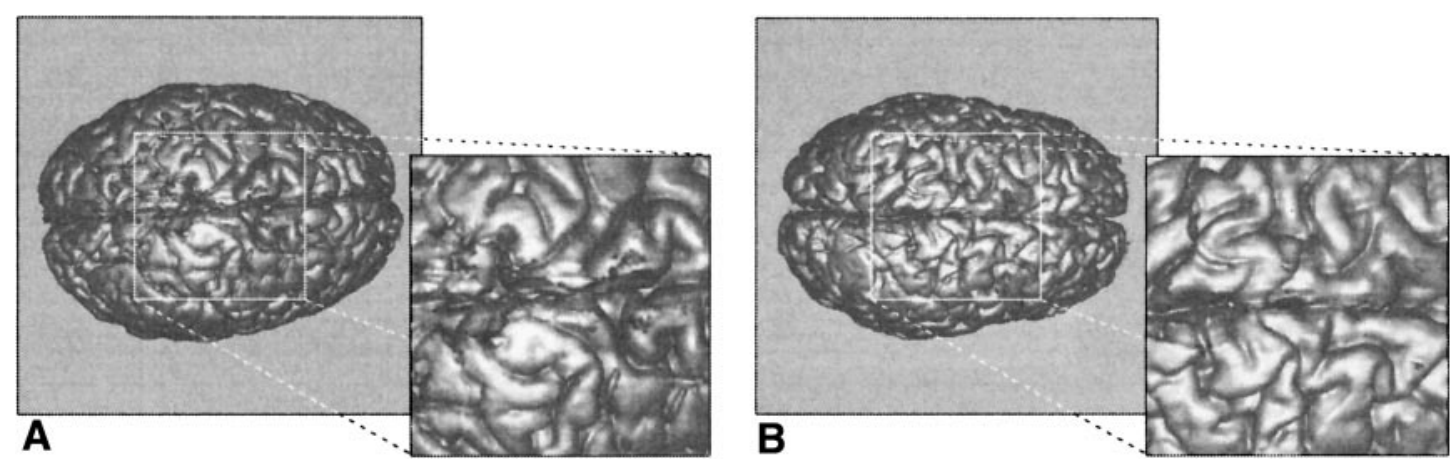

FIG. 13. Volume renderings of MBRASE segmentations for two patient sets with an enlargement. Frame (A) shows results for patient dataset XXI (average similarity), and Frame (B) patient dataset XXIX (highest similarity). Both renderings contain an enlargement of the Iongitudinal cerebral fissure. The differences between these renderings and the renderings of their respective reference datasets are hardly noticeable; accordingly the latter renderings are not shown. 
Segmentation of a typical dataset requires about half an hour of CPU time on a processor of an SGI Power Challenge R10K (MIPS R10000, $195 \mathrm{MHz}$ ). Speed of segmentation was not considered a main issue for this work, but it can be considerably improved by optimizing the implementation and/or using the inherent parallelism of the algorithm.

Others have previously reported on fully automatic brain segmentation from MR image data. Collins et al. (1995a) use an approach where the transformation between a model brain and a new MRI brain volume is first determined in a registration step. The segmentation is then performed by applying the transformation to map atlas contours from the model brain to the new MRI brain volume. The results are very encouraging, but the authors express caution by stating that the required 1-to-1 correspondence between model and MRI brain is never strictly true because of normal morphological variability and the influence of pathology. Other fully automatic segmentation techniques (Brummer et al., 1993; Saeed et al., 1997; Atkins and Mackiewich, 1998; Lemieux et al., 1999) have used algorithms that are basically chains of separate procedures to refine the segmentation of the brain in each subsequent step. Unfortunately, this renders the algorithms complex (especially for software encoding) and a multitude of parameter values have to be set. Our objective was to automate the critical observer decisions in an already established and simple method. The results are a strong indication that we were successful in replacing these critical decisions using simple procedures with few parameters. The fact that our method is primarily based on just one critical decision, i.e., detection of the connection(s) of the brain with other structures, is potentially a weakness, but has been turned into a strength by restricting its application to a specific and well defined task. The result is a simple segmentation method with surprisingly good results.

\section{CONCLUSIONS}

An automated approach has been developed to segment the brain from T1-weighted MR images. This method, denoted MBRASE, is based on the simple operations thresholding, erosion, and geodesic dilation.

Computer simulated data have been used to evaluate the segmentation performance against a gold standard. The results are found to be both robust and in excellent agreement with the reference data. Supervised brain segmentations of the MRI-T1 data of 30 patients, carried out by a neuroanatomy expert, have been used to test the performance of MBRASE on clinical data. An objective comparison shows that the results of the automated segmentation are quite similar to the supervised segmentations. Brain volume visualizations based on the automated and supervised seg- mentations are generally visually indistinguishable. In consequence, the proposed method can be used as a default segmentation for quantitation and visualization of the human brain from T1-weighted MR images in routine clinical procedures.

\section{ACKN O WLEDG MENTS}

We are indebted to our colleagues W. F. C. Baaré, R. Maas, Dr. L. C. Meiners, Dr. W. J . Niessen, Dr. H. G. Schnack, Dr. E. P. A. Vonken, and R. van der Weide. The Radiology Department of the Academic Hospital Utrecht kindly provided the MR image data. We gratefully acknowledge the research license of ANALYZE, provided by Dr. R. A. Robb, Mayo Foundation/Clinic, Rochester, Minnesota.

\section{REFERENCES}

Adams, R., and Bischof, L. 1994. Seeded region growing. IEEE Trans. Pattern Anal. 16(6): 641- 646.

Atkins, M. S., and Mackiewich, B. T. 1998. Fully automatic segmentation of the brain in MRI. IEEE Trans. Med. Imag. 17(1): 98-107.

Balm, R., Stokking, R., Kaatee, R., Blankensteijn, J . D., Eikelboom, B. C., and van Leeuwen, M. A. 1997. Computed tomographic angiographic imaging of abdominal aortic aneurysms: Implications for transfemoral endovascular aneurysm management. J . Vasc. Surg. 26(2): 231-237.

Barillot, C., Lachmann, F., Gibaud, B., and Scarabin, J . M. 1991. 3-D display of MRI data in neurosurgery: Segmentation and rendering aspects. In SPIE Medical Imaging V: Image Processing (M. H. Loew, Ed.), Vol. 1445, pp. 54-65. SPIE.

Brummer, M., Mersereau, R. M., Eisner, R. L., and Lewine, R. R. J . 1993. Automatic detection of brain contours in MRI data sets. IEEE Trans. Med. Imag. 12(2): 153-166.

Chang, Y.-L., and Li, X. 1994. Adaptive image region-growing. IEEE Trans. Imag. Proc. 3(6): 868-872.

Clarke, L. P., Velthuizen, R. P., Camacho, M. A., Heine, J . J ., Vaidyanathan, M., Hall, L. O., Thatcher, R. W., and Silbiger, M. L. 1995. MRI segmentation: Methods and applications. Magn. Reson. Imag. 13(3): 343-368.

Cline, H. E., Dumoulin, C. L., Hart, J r., H. R., Lorensen, W. E., and Ludke, S. 1987. 3D reconstruction of the brain from magnetic resonance images using a connectivity algorithm. Magn. Reson. Imag. 5: 345-352.

Cocosco, C. A., Kollokian, V., Kwan, R. K.-S., and Evans, A. C. 1997. Brainweb: Online interface to a 3-D MRI-simulated brain database. Neurol mage 5(4): S425. Proceedings of 3rd International Conference on Functional Mapping of the Human Brain, Copenhagen.

Collins, D. L., Evans, A. C., Holmes, C. J ., and Peters, T. M. 1992. Model based segmentation of individual brain structures from MRI data. In Visualization in Biomedical Computing (R. A. Robb, Ed.), pp. 10-23, SPIE Press, Bellingham, WA.

Collins, D. L., Evans, A. C., Holmes, C. J ., and Peters, T. M. 1995a. Automatic 3D segmentation of neuro-anatomical structures from MRI. In Information Processing in Medical Imaging (Y. Bizais, C. Barillot, and R. di Paola, Eds.), pp. 139-152. Kluwer Dordrecht.

Collins, D. L., Holmes, C. J ., Peters, T. M., and Evans, A. C. 1995b. Automatic 3-D model-based neuroanatomical segmentation. Hum. Brain Map. 3: 190-208.

Collins, D. L., Zijdenbos, A. P., Kollokian, V., Sled, J ., Kabani, N. J ., Holmes, C. J ., and Evans, A. C. 1998. Design and construction of a realistic digital brain phantom. IEEE Trans. Med. I mag. 17: 463468. 
Dale, A. M., Fischl, B., and Sereno, M. I. 1999. Cortical surface-based analysis. Neurol mage 9(2): 179-194.

Evans, A. C., Collins, D. L., and Holmes, C. J . 1995. Automatic 3D regional $\mathrm{MRI}$ segmentation and statistical probability anatomy maps. In Quantification of Brain Function: Tracer Kinetics and Image Analysis in Brain PET (T. J ones, Ed.), pp. 123-130. Academic Press.

Gerig, G., Martin, J ., Kikinis, R., Kübler, O., Shenton, M. E., and J olesz, F. A. 1992. Unsupervised tissue type segmentation of 3D dual-echo MR head data. I mage Vision Comput. 10(6): 349-360.

Höhne, K. H., and Hanson, W. H. 1992. Interactive 3D segmentation of MRI and CT volumes using morphological operations. J . Comput. Assist. Tomogr. 16(2): 285-294.

J iang, H., Toriwaki, J ., and Suzuki, H. 1993. Comparitive performance evaluation of segmentation methods based on region growing and division. Syst. Comput. J ap. 24(13): 28- 42.

J ustice, R. K., Stokely, E. M., Strobel, J . S., I deker, R. E., and Smith, W. M. 1997. Medical image segmentation using 3-D seeded region growing. In Medical Imaging, Vol. 3034, Proc. SPIE, pp. 900-910. SPIE Press, Bellingham, WA.

Kikinis, R., Shenton, M. E., Gerig, G., Martin, J ., Anderson, M., Metcalf, D., Guttmann, C. R. G., McCarley, R. W., Lorensen, W. E., Cline, H. E., and J olesz, F. A. 1992. Routine quantitative analysis of brain and cerebrospinal fluid spaces with MR imaging. J . Magn. Reson. Imag. 2: 619-629.

Kohn, M. I., Tanna, N. K., Herman, G. T., Resnick, S. M., Mozley, P. D., Gur, R. E., Alavi, A., Zimmerman, R. A., and Gur, R. C. 1991. Analysis of brain and cerebrospinal fluid volumes with MR imaging; part I. methods, reliability, and validation. Radiology 178: 115- 122.

Kwan, R. K. S., Evans, A. C., and Pike, G. B. 1999. MRI simulationbased evaluation of image-processing and classification methods. IEEE Trans. Med. Imag. 18(11): 1085-1097.

Lemieux, L., Hagemann, G., Krakow, K., and Woermann, F. G. 1999. Fast, accurate, and reproducible automatic segmentation of the brain in T1-weighted volume MRI data. Magn. Reson. Med. 42: 127-135.

Levoy, M. 1988. Rendering of surfaces from volume data. IEEE Comput. Graph. 8(3): 28-37.

Niessen, W. J . 1997. Multiscale Medical Image Analysis. PhD thesis, Utrecht University, the Netherlands.

Robb, R. A., and Hanson, D. P. 1996. The ANALYZE software system for visualization and analysis in surgery stimulation. In Computer-I ntegrated Surgery (R. H. Taylor, S. Lavallée, G. C. Burdea, R. Mösges, Eds.), pp. 175-189. MIT Press, Cambridge, MA.

Saeed, N., Hajnal, J . V., and Oatridge, A. 1997. Automated brain segmentation from single slice, multislice, or whole-volume MR scans using prior knowledge. J . Comput. Assist. Tomogr. 21(2): 192-201.
Sandor, S., and Leahy, R. M. 1995. Toward automated labeling of the cerebral cortex using a deformable atlas. In Proceedings I nformation Processing in Medical Imaging (Y. Bizais, C. Barillot, and R. di Paola, Eds.), pp. 127-138. Kluwer, Dordrecht.

Sekiguchi, H., Sano, K., and Yokoyama, T. 1994. Interactive 3-dimensional segmentation method based on region growing method. Syst. Comput. J ap. 25(1): 88-97.

Shenton, M. E., Kikinis, R., J olesz, F. A., Pollack, S. D., LeMay, M., Wible, C. G., Hokama, H., Martin, J., Metcalf, D., Coleman, M., and McCarley, R. W. 1992. Abnormalities of the left temporal lobe and thought disorder in schizophrenia. New Engl. J. Med. 327: 604- 612 .

Sled, J . G., Zijdenbos, A. P., and Evans, A. C. 1998. A nonparametric method for automatic correction of intensity nonuniformity in MRI data. IEEE Trans. Med. Imag. 17: 87-97.

Stokking, R. 1998. Integrated Visualization of Functional and Anatomical Brain Images. PhD thesis, Utrecht University, the Netherlands.

Stokking, R., van Isselt, J. W., van Rijk, P. P., de Klerk, J . M. H., Huiskens, A. W. L. C., Mertens, I. J. R., Buskens, E., and Viergever, M. A. 1999. Validation of techniques for integrated visualization of functional and anatomical brain data. J. Nud. Med. 40(2): 311-316.

Stokking, R., Studholme, C. S., Spencer, S. S., Avery, R. A., Corsi, M., Eickel berg, C., Morano, G. N., Seibyl, J . P., and Zubal, I. G. 2000. Epilepsy related perfusion changes in the peripheral cortex. J. Nud. Med. 41(5): 65P-66P.

Van den Elsen, P. A. 1993. Multimodality Matching of Brain I mages. PhD thesis, Utrecht University, the Netherlands.

Velthuizen, R. P., Heine, J . J ., Cantor, A. B., Lin, H., Fletcher, L. M., and Clarke, L. P. 1998. Review and evaluation of MRI nonuniformity corrections for brain tumor response measurements. Med. Phys. 25(9): 1655-1665.

Vincken, K. L., Niessen, W. J ., and Viergever, M. A. 1998. Subvoxel segmentation of MR images: Necessity and approach. In Sixth Scientific Meeting and Exhibition, Proceedings of the International Society for Magnetic Resonance in Medicine, p. 2070. ISMRM.

Wells, III, W. M., Grimson, W. E. L., Kikinis, R., and J olesz, F. A. 1996. Adaptive segmentation of MRI data. IEEE Trans. Med. Imag. 15(4): 429-442.

Zijdenbos, A., Dawant, B. M., Margolin, R. A., and Palmer, A. C. 1994. Morphometric analysis of white matter lesions in MR images. IEEE Trans. Med. Imag. 13(4): 716-724.

Zucker, S. W. 1976. Region growing: Childhood and adolescence. Comput. Graph. Imag. Process. 5: 382-399.

Zuiderveld, K. J . 1995. Visualization of Multimodality Medical Volume Data Using Object-Oriented Methods. PhD thesis, Utrecht University, the N etherlands. 